\title{
Factores que inciden en el desarrollo rural en la comunidad Las Perlas, Ticuantepe, 2014
}

\author{
José Ramón Velásquez Hernández
}

Recepción: 30 - 10 - 2015 / Aceptación: 03 - 12 - 2015

\section{Resumen}

En esta investigación se analizaron los factores socioeconómicos y culturales que inciden en el desarrollo rural de la comunidad de Las Perlas, municipio de Ticuantepe. La premisa de que se parte se sustenta en que las variantes culturales como: la educación, las relaciones de género, la cosmovisión, los patrones culturales de producción, moldean el comportamiento del productor e inciden en sus prácticas productivas. La metodología utilizada fue cualitativa, se aplicaron encuestas para obtener información sobre cada variable, y entrevistas que permitieron reforzar la información. Entre los principales resultados de los factores socioeconómicos, está la educación, donde el 86.7\% tienen educación primaria y secundaria, y ninguno con educación superior, el salario percibido por los productores es de 3,000 y está por debajo de la media nacional que es de 3,448 córdobas. El 80\% de las familias tienen una composición familiar entre dos y cuatro personas, por tanto el ingreso económico mensual no logra satisfacer las necesidades básicas. Ninguna mujer es dueña de la tierra, lo que acentúa las disparidades de género, el 80\% de los pobladores no pertenecen a ninguna organización, solamente el $20 \%$ pertenecen a organizaciones politicas. Se concluye que la incipiente organización, la tenencia de la tierra, los bajos ingresos y el aspecto politico institucional inciden en el desarrollo rural de la comunidad.

Palabras clave: Cultura, patrón cultural, unidad doméstica de producción, factores socioeconómicos, cosmovisión, visión cultural del desarrollo.

\begin{abstract}
In this research, socioeconomic and cultural factors that influence the development of the rural community of Las Perlas, Ticuantepe analyzed. This research was conducted because cultural variations such as education, gender relations, worldview, the cultural patterns of production, the producer shape behavior and affect their production practices. The methodology was qualitative surveys were applied to obtain information about each variable, and interviews allowed strengthening the information. The main results of socioeconomic factors is education, where $86.7 \%$ have primary and secondary education, higher education and none, the salary received by producers is 3,000 and is below the national average of 3,448 cordobas . $80 \%$ of families have a family composition between two and four people, so the monthly income fails to meet basic needs. No woman owns land, exacerbating gender disparities, $80 \%$ of people do not belong to any organization, only $20 \%$ belong to political organizations. It is concluded that the fledgling organization, land tenure, low income and institutional political aspect affecting rural community development.
\end{abstract}

Keywords: Culture, cultural pattern, household production, socioeconomic factors, worldview, cultural vision of development 


\section{Introducción}

Las investigaciones realizadas en la sociedad rural, tradicionalmente han estado enfocadas en dos aspectos: agronómicos y económicos. Sin embargo algunos estudios clásicos como Wolf (1971) un clásico de la Antropología Rural, estudia desde la cultura aspectos funcionales del campesinado, en tal estudio se incluyen tres aspectos macro: lo social, lo económico y lo ideológico de la cultura campesina. También Fromm y Maccoby (1990) abordan al campesino mexicano desde el punto de vista psicológico y sociológico, enfatizando en la conducta social y en características socioeconómicas.

En los últimos años, se han venido fortaleciendo las investigaciones que relacionan el desarrollo rural con la cultura, un estudio realizado por Matijasevic, MT (2007) sobre los significados atribuidos al desarrollo rural de la Vereda, municipio de Manizales, en Colombia, se exploran los significados que los pobladores y profesionales atribuyen al desarrollo rural, utilizando la metodología cualitativa para comprender la construcción social del concepto desde un contexto particular.

Un estudio realizado por Jaramillo, LF (2007) denominada: Elementos para el análisis de la población rural en la zona centro de Urabá, Colombia, versa sobre: el análisis de tres poblaciones rurales en donde se destacan las características del grupo cultural, localización en el territorio y actividad económica, el estudio de acuerdo a la autora, también aborda el análisis en cuanto a su estructura agraria, sistema agroindustrial y campesinado. Este estudio de la cuestión rural también integra elementos etnográficos como metodología y antropológicos fundamentalmente en cuanto a su teorización y conceptualización.

Siempre en el marco de esta relación cultura y desarrollo rural, Bonatti, $\mathrm{M}(2007)$, en su estudio sobre cambios climáticos, percepciones humanas y desarrollo rural, analiza la forma en como las poblaciones humanas de Anchieta, Brasil, entienden el cambio climático desde sus percepciones, partiendo en cómo las comunidades entienden un fenómeno determinado y qué efectos tiene sobre sus prácticas agrícolas, pero que además todo esto es dado como una estrategia adaptativa que parte de la cultura misma, la autora hace énfasis en cómo los estudios de percepción son relevantes para los programas de desarrollo.

Es necesario también mencionar el estudio realizado en Cuba por Cino, DM(2009) sobre el desarrollo rural, social y económico, suscitado con la introducción del ganado bubalino, en donde destaca los cambios socioeconómicos y culturales ocurridos durante este proceso, donde además de hacer una caracterización socioeconómica del sector estudiado, aborda las percepciones que lo actores sociales involucrados tienen sobre la influencia del ganado bubalino en el sector ganadero, y la forma en esta nueva especie impacta en el desarrollo rural cubano. Este trabajo por sus características también constituye un antecedente de la investigación presentada en este trabajo.

Un estudio más contextual es el realizado por Romero, J, et al.(2009) sobre Ticuantepe y su Historia; versa sobre lo histórico que transversaliza en el análisis del potencial arqueológico, la geografía del municipio, el uso de la tierra, la división política administrativa, la economía, los servicios básicos, la educación, lo político y lo cultural. Este estudio es el más cercano que existe en cuanto a estudios realizados en el contexto, aplicando metodologías de análisis social cualitativo como entrevistas e historias de vida.

Es relevantey pertinenteen esta investigación, hacer mención de los estudios realizados por Serra, L (1991) sobre El movimiento Campesino: su participación política durante la revolución Sandinista, donde establece primeramente categorías conceptuales 
elaboradas por el autor y otras retomadas de otros autores sobre el campesinado, además de analizar el movimiento político campesino, sus formas de organización social y su papel en la revolución. También Serra (1987) realizó un trabajo científico sobre los Aportes para una conceptualización del campesinado. Estos estudios realizados por este autor son de suma importancia para el estudio realizado en Las Perlas porque permite un acercamiento teórico en contexto nacional, además porque se trata de una comunidad rural compuesta por campesinos de acuerdo a las características culturales que esta tiene.

El CIPRES (2006) en un estudio sobre los pequeños y medianos productores, hacen un abordaje desde el punto de vista social, económico, productivo y agronómico, pero no hay un estudio profundo sobre la cultura del productor y su incidencia en el desarrollo comunitario.

Ticuantepe, es un municipio del departamento de Managua, ubicado a 18 kilómetros de la capital, en él se encuentra ubicada Las Perlas comunidad rural de 850 habitantes, contexto social donde se analizan los factores socioeconómicos y culturales que inciden en el Desarrollo Rural, en esta temática se aborda el comportamiento cultural de los sujetos rurales desde el punto de vista su dinámica de vida rural. El análisis de la cultura no solamente es importante porque estudia los factores endógenos que estimulan determinado comportamiento social, sino que permite saber las causas y consecuencias de determinados comportamientos sociales y patrones culturales que tienen incidencia en el desarrollo rural de la comunidad.

En el año 2011, una de las instituciones encargadas del desarrollo rural, el INTA, con sede en el municipio de Ticuantepe, y la alcaldía municipal, estaban interesados en fortalecer las alianzas con la comunidad, y para este propósito se hacía necesario comprender aquellos aspectos socioeconómicos y culturales que tenían incidencia en el desarrollo rural de la comunidad.

En el marco de estos propósitos institucionales surge la necesidad de emprender esta tarea investigativa. Porque todo proceso de desarrollo rural debe tener como eje transversal el aspecto políticoinstitucional, pasando por el reconocimiento de la cultura comunitaria rural y de los aspectos socioeconómicos que caracterizan la vida rural comunitaria, lo que conlleva a plantear el siguiente problema: ¿Qué factores socioeconómicos y culturales inciden el desarrollo rural de la comunidad de Las perlas?

Por consiguiente el desarrollo rural se analiza desde los factores culturales como la educación, término que también hace referencia al proceso de construcción social de las personas desde lo interno de las familias y luego en el entorno social, la cultura es una concepción amplia que implica el tipo de religión, los ciclos en que se cultivan determinados rubros, los aspectos mitológicos sobre la siembra, la identidad y cultura productiva, la composición y tamaño de la familia, el tipo de tecnología utilizada, la forma de organizar el trabajo entre otros aspectos propios de su dinámica cultural.

El Desarrollo Rural también se puede analizar desde los aspectos socioeconómicos como el acceso a la tierra, el tamaño de la parcela, el ingreso económico familiar, los costos de insumos, las fuentes de financiamiento para la producción y el tipo de asistencia técnica que se les pueda brindar a los productores de la comunidad.

Se establecen relaciones intrínsecas entre aspectos económicos y culturales, como el acceso a la tierra que puedan tener hombres y mujeres que involucra un fenómeno de relaciones de género. Las percepciones sobre lo rural se interpretan en base a la información proporcionada por los comunitarios. 
La construcción de cultura económica desde los productores, obedece a la configuración de determinados patrones culturales que se transmiten de forma intergeneracional, en donde los procesos de aprendizaje en torno a lo socio-cultural y lo económico son dados por el entorno y por las relaciones sociales comunitarias, esto quiere decir que los conocimientos en la agricultura o bien en otras actividades económicas que tiene las unidades domésticas de producción se van aprendiendo por la transmisión oral de padres a hijos.

Esta investigación es importante porque brinda la posibilidad de estudiar el desarrollo Rural desde la cultura de los pobladores, para complementar los estudios agronómicos y socioeconómicos que se han realizado sobre el desarrollo rural.

En términos sociales se hace un aporte científico desde el análisis las causas culturales y socioeconómicas en que inciden en el comportamiento de la población en relación al desarrollo rural, y finalmente puede contribuir a mejorar la calidad de vida.

\section{Metodología}

En este apartado se detalla todo el proceso metodológico llevado a cabo durante el trabajo de campo realizado durante los años 2011, 2012, 2013, para la recopilación de información, utilizando encuestas, entrevistas y observaciones para hacer un reconocimiento pertinente de la realidad social. Se abordan desde las concepciones filosóficas del método, pasando por cada una de las fases del proceso investigativo, que incluye la selección de la muestra teórica, hasta la matriz de operacionalización de variables y matriz de descriptores. De manera que esta investigación cuenta de fases y pasos a seguir para la consecución de los objetivos propuestos, todo esto define un proceso, que se describe a continuación:

\section{Etapas del proceso investigativo}

Fundamentos filosóficos del Método: La perspectiva de investigación que se utilizó fue cualitativa. El tipo de investigación es el no experimental, porque este sentido el investigador no manipula variables, sino que las variables sociales, culturales y económicas ya están establecidas. Además es descriptiva porque se estudian comportamientos y se comprueban asociaciones entre las variables estudiadas. Se utilizaron encuestas y entrevistas como técnicas para la recopilación de la información. De acuerdo al diseño metodológico es descriptivo. Según el método de estudio es observacional (Piura, 2006). De acuerdo, al tiempo de ocurrencia de los hechos y registro de la información, el estudio es prospectivo y según el período y secuencia del estudio es transversal, (Canales, Alvarado y Pineda, 1996).

\section{Fase I. Fase Preparatoria}

Paso 1. Para esta investigación se ubicó el problema: ¿Qué factores socioeconómicos y culturales inciden el desarrollo rural de la comunidad de Las perlas?

Paso 2. La selección de la comunidad Las Perlas, ubicada geográficamente en el municipio de Ticuantepe, se decide porque fue una prioridad del Instituto Nicaragüense de Tecnología Agropecuaria (INTA) y de la alcaldía municipal para el establecimiento de parcelas de validación, y para la construcción de una base social que permitiera el trabajo en conjunto entre técnicos y productores. Además presentaba todas las características sociales, económicas y productivas para la realización del estudio, y se establecieron previamente los contactos con líderes comunitarios para la realización del estudio. En la ubicación del área de estudio se selecciona la comunidad tomando en consideración los siguientes criterios: 1 . La agricultura diversificada en la comunidad. 2. Es de fácil acceso tanto en términos económicos como geográficos. 3 . El contexto 
social fue propicio para el desarrollo de la investigación. 4. Presencia de actores políticos-institucionales. 5. Organización comunitaria consolidada 6. En la comunidad seleccionadas hay productores clasificados entre pequeños y medianos.

Paso 3: Selección de los informantes Los criterios para seleccionar a los informantes (muestreo cualitativo) fueron los siguientes: 1.El poblador seleccionado trabaja en agricultura o en otra actividad económica. 2. La parcela debe estar ubicada en la comunidad. 3. El comunitario al menos debe tener cinco años de residir en la comunidad. 4. El comunitario debe estar activo en sus labores agrícolas o bien en otra actividad económica. 5. El tamaño de la parcela debe tener como mínimo $0.35 \mathrm{Ha}$ equivalente a $0.5 \mathrm{mz}$. 6 . Deben ser hombres y mujeres entre 16 y 80 años.

La muestra cualitativa fue de nueve entrevistados, porque las muestras cualitativas no son utilizadas para representar a una población, sino para comprender el significado que tienen los actores sociales sobre un fenómeno en particular. El tipo de muestra cualitativa se denomina de acuerdo a Hernández, et al (2006) muestra diversa o de máxima variación, en donde en el caso concreto, de las concepciones del desarrollo rural, muestran diversas perspectivas sobre el tema estudiado, y se seleccionaron además de agricultores, a jóvenes que realizan otro tipo de trabajos como cuidar fincas y a mujeres que son dueñas de negocios familiares como pulperías.

También de acuerdo con Munch, L(1996), obedece a un tipo de muestreo no probabilístico de tipo decisional, en donde los investigadores de campo utilizan su criterio para seleccionar los elementos de la muestra. La muestra obtenida para aplicar las encuestas fue de 15 productores y productoras. El tipo de muestra utilizada es no probabilístico por conveniencia, Hernández, et al (2006) estos encuestados constituyen informantes a los que se tuvo acceso en el momento de aplicar el instrumento.

Se prepararon las técnicas de encuestas y entrevistas y se validaron los instrumentos, posteriormente se realizó trabajo de campo, y se aplicaron las técnicas validadas a las familias de la comunidad. En el año 2011 se realizó un recorrido por la comunidad y se hicieron observaciones generales, y entrevistas preliminares para conocer y contactar a líderes locales, en el año 2012 se aplicaron encuestas como instrumentos de recolección de información, en el año 2013 se procesaron los datos cualitativos a través de matrices organizadores, y los datos cuantitativos a través del SPSS versión 18, luego la fase analítica de análisis y estructuración de la información y posteriormente la realización del informe.

\section{Resultados de Investigación}

\section{Escolaridad de los productores}

La escolaridad es uno de los indicadores abordados en este estudio, además es un indicador de desarrollo humano. A partir del nivel de escolaridad se pueden visualizar las oportunidades de desarrollo en una comunidad. El $46.7 \%$ de los productores y productoras tienen educación primaria, es decir educación básica, mientras que el 40\% tienen educación secundaria este segmento de población tiene mejores expectativas de desarrollo desde las familias, y un $6.7 \%$ tienen estudios técnicos, y otro $6.7 \%$ no puede leer ni escribir. La educación formal en la comunidad necesita fortalecimiento, por los datos antes mencionados, por consiguiente esta ventana al desarrollo es estrecha, porque limita las oportunidades. 
Figura 1. Porcentaje de escolaridad de los productores.

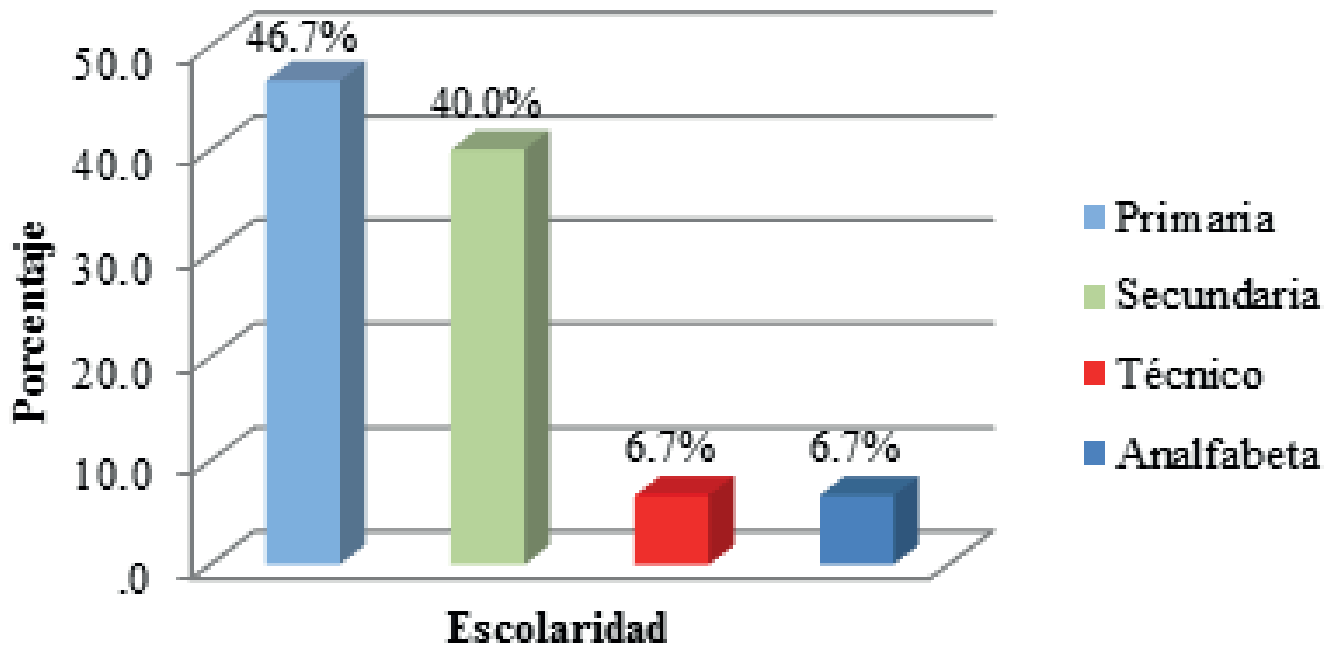

Fuente propia

En un estudio realizado por Vargas, $\mathrm{O}$ (2005) expresa que: la educación primaria representa la piedra angular de las oportunidades educativas y forma parte del compromiso constitucional que el estado tiene con los nicaragüenses. De la población entre 15 y 45 años, el 36\% de la población no posee primaria completa.

Esto significa que la realidad de la comunidad de Las Perlas con el $46.7 \%$ de productores con educación primaria, está por encima de la media nacional de ese año que representaba el $36 \%$ de la población, esto hace denotar que hay una realidad nacional que se vincula con realidad educativa de la comunidad rural.

En relación a este aspecto (Chavarría, 2013) expresa: "Hay un colegio preescolar y primaria matutino, pero para estudiar la secundaria hay que ir a Ticuantepe a los colegios: Manuel Lández y Pedro Joaquín Chamorro. Sería bueno un colegio de secundaria pero la comunidad estudiantil es pequeña, los que entran a secundaria son como máximo 50 estudiantes y por lo tanto no sería una inversión rentable".
En la comunidad solamente existe una escuela, que brinda educación preescolar y primaria, sin embargo no hay educación secundaria, los estudiantes tienen que viajar hasta el casco urbano del municipio de Ticuantepe para poder estudiar, pero este no puede ser considerado como un factor que contribuya al bajo nivel académico en la comunidad, en contextos rurales tal y como se ha expresado en acápites anteriores, los jóvenes tienen como patrón de comportamiento social, trabajar en vez de estudiar porque esta representa una inversión al largo plazo, y lo que ellos necesitan es resolver sus necesidades de día a día.

\section{Tipo de trabajo desempeñado}

Las familias de productores encuestadas durante el estudio, tienen variantes en cuanto a su actividad económica, es decir que no solamente se dedican a la agricultura, sino que trabajan como comerciantes y guardas de seguridad, esto no significa que las dos últimas actividades económicas sustituyan a la primera, sino que en el contexto comunitario son complementarias. 
Figura 2. Porcentaje de tipo de trabajo desempeñado por los productores.

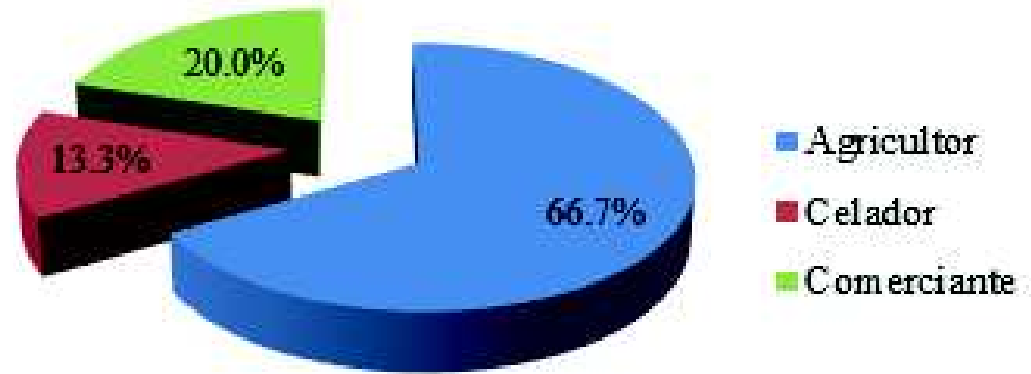

Fuente propia

En Las Perlas el $66.7 \%$ se dedican exclusivamente a la agricultura, uno de los problemas de la pobreza rural, es que los pobladores solamente tengan la vía agrícola como única opción. El $20 \%$ trabajan como comerciantes, pero ésta es una actividad complementaria a la agricultura, de igual manera sucede con $13.3 \%$ que son guardas de seguridad.

En tanto De Janvry y Sadoulet (2000) plantean que la inversión en el activo del campesinado pobre puede ayudarlo a salir de la pobreza por una multiplicidad de caminos: la emigración, la vía agrícola, la pluriactividad y la vía asistencial. En relación al tipo de trabajo que desempeñan los comunitarios, el señor (Chavarría, 2013) argumenta: “Los tipos de trabajo son: agricultura, construcción, textilerías, guardas de seguridad; las personas que trabajan en las tres últimas actividades mencionadas salen a Managua.

Con el tipo de trabajo lo que hace la gente es sobrevivir" (ver anexo 1). En la comunidad, si bien la agricultura constituye el eje central de la dinámica económica, se encuentran otras actividades como: la vigilancia privada, la construcción, los pequeños negocios entre otros, esto apunta hacia la pluriactividad que plantean los autores arriba señalados, de manera que las familias buscan la forma de diversificar sus actividades productivas o bien trabajar en una actividad que no sea la agricultura para obtener un ingreso económico.

\section{Ingresos Familiares}

Los ingresos familiares en la comunidad, reflejan de manera directa las condiciones de vida de sus habitantes y por lo tanto representa un indicador socioeconómico que incide en el desarrollo rural.

Figura 3. Porcentaje de ingresos familiares. 
El 53\% de los productores de la comunidad tienen un ingreso de 3,000 córdobas mensuales, este ingreso está por debajo de del salario mínimo establecido en Nicaragua, de acuerdo a las negociaciones salariales de septiembre de 2012, (MITRAB(2012) que alcanzó los 3, 448 córdobas (promedio general).

Sin embargo la estructura del salario mínimo es por sector, y para el agropecuario es de 2,273.80, lo que significa que está por encima de lo establecido para este sector. Otro segmento de productores de la comunidad de Las Perlas percibe 2,000 córdobas mensuales, con una diferencia 1,448 córdobas mensuales por debajo del salario mínimo general y 2,73.70 córdobas menos que el establecido para el sector agropecuario.

Un segmento del13.3\% alcanza 4,000 córdobas mensuales y otro del mismo peso porcentual (13.3\%) logra percibir 5,000 córdobas, por encima del salario mínimo establecido, son las familias de acuerdo a las observaciones realizadas tiene mejores condiciones de vida. Sin embargo de acuerdo al INIDE (2013), la canasta básica a marzo del 2013 es de 10,784 córdobas, distribuida de la siguiente manera:

Cuadro 1. Distribución de ingresos de la canasta básica

$\begin{array}{cccc}\begin{array}{c}\text { Alimentos } \\ \text { básicos }\end{array} & \begin{array}{c}\text { Usos del } \\ \text { hogar }\end{array} & \text { Vestuario } & \text { Total } \\ 6,887.16 & 2,659.39 & 1,238.13 & 10,784.78\end{array}$

Fuente IPC-BCN

Esto indica que el $100 \%$ de los productores y productoras de la comunidad, no logran satisfacer completamente sus necesidades prioritarias porque sus ingresos están muy por debajo de la canasta básica, y ni siquiera logran cubrir los alimentos básicos porque se requiere de 6,887.16 córdobas. El 73\% de los productores tiene ingresos que oscilan entre
2,000 y 3,000 córdobas, y otro segmento que representan el $26,6 \%$ tiene ingresos entre 4,000 y 5,000 córdobas mensuales.

El ingreso familiar es un factor que incide significativamente en las condiciones $y$ calidad de vida que tienen las familias, y esto es el reflejo de las condiciones de pobreza en la comunidad. Desde el punto de vista cuantitativo (ingresos), este factor tiene incidencia en el desarrollo comunitario porque los indicadores de salario mínimo y de la canasta básica, no son alcanzados por los productores y productoras.

La unidad doméstica de producción no compra lo que no necesita, esto forma parte de su racionalidad económica, y no estarían en disposición, ni necesitarían adquirir los 53 productos que contiene la canasta básica, pero en definitiva si se compara con este parámetro los efectos para el desarrollo rural de la comunidad son adversos. Lo que está claro es que las familias de la comunidad están buscando estrategias como: la diversificación productiva, la pluriactividad económica, para incrementar sus ingresos económicos y tener mayor poder adquisitivo, que por ende derivaría en un mejoramiento de sus condiciones de vida.

\section{Tenencia de la tierra}

Este es un componente relacionado con un factor socioeconómico, en la medida en que los productores tienen una forma particular de adquirir la propiedad, desde el punto de vista de su situación jurídicolegal y económico en la tenencia de la tierra, también es un asunto cultural, en cuanto hay inequidad en cuanto al género cuando se adquiere o se posee este factor de producción. La comunidad estudiada antes era una finca de acuerdo a información de los pobladores, y empezó a formarse socioculturalmente, en década de 1980 en el contexto de la reforma agraria, de manera que un fenómeno políticoinstitucional y de relevancia histórica contribuyó a su fundación. 
Las propiedades y la tenencia de la tierra están fundamentadas en este hecho socioeconómico. En la realidad encontrada a nivel doméstico, el $40 \%$ de los productores son dueños de su parcela, lo que garantiza la utilización libre de los recursos naturales de acuerdo a sus necesidades y aspiraciones, un productor que es dueño de sus medios de producción tiene mejores oportunidades para mejorar sus condiciones de vida.

El 33.3\% de los productores prestan la tierra, aunque este es un acto de cooperación y solidaridad, de quienes poseen este medio de trabajo hacia quienes no lo tienen, y deja en evidencia relaciones de reciprocidad como un elemento fuerte del capital social.

Esta situación es un indicador que puede estar incidiendo negativamente en el desarrollo familiar y comunitario, porque las familias al carecer de este medio de producción no pueden hacer mejoras en la parcela, ni definir y establecer estrategias productivas para mejorar sus ingresos porque está a expensas de la voluntad del dueño.
Un $26.7 \%$ de los productores rurales tienen que alquilar tierras para trabajar en las labores agrícolas, las desventajas en relación al grupo que presta son similares, porque no puede diversificar cultivos o incorporar mejoras, sin embargo el primero basa sus relaciones en la reciprocidad, en la confianza como elemento clave del capital social. En el último caso la relación es utilitaria, basada además en un precio que se adquiere por rentar la tierra, y estos ingresos ya no tienen como paradero las estructuras familiares del productor desposeído, sino que se destinan al propietario de la tierra.

Este fenómeno no permitirá el desarrollo socio económico, cultural y productivo de las familias y de las comunidades, porque el $60 \%$ de los productores no tienen o no cuentan con tierra propia, por los que la alquilan o la prestan. Este es un factor socioeconómico y cultural de significancia que limita el desarrollo rural.

Figura 4. Porcentaje de la tenencia de la tierra.

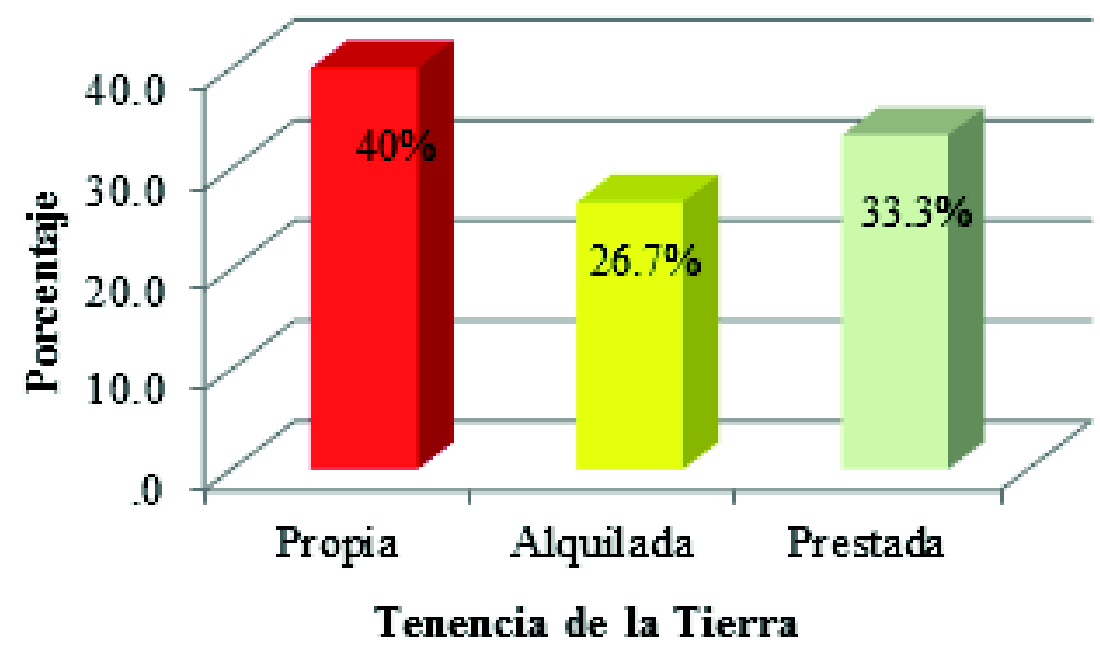

Fuente propia 
El prestar la tierra a otros productores, obedece a un asunto cultural, porque se tejen redes y relaciones sociales, al contrario el que renta la tierra tiene una visión economicista y de beneficio individualista. En un estudio realizado por Mansky (1997) considera que: "La tenencia de la tierra es un factor que influye significativamente en la adopción de nuevas tecnologías, (variedades), y se considera que hay una respuesta positiva en tierra propia y posibilidades de adopción".

La tierra es elemental para los procesos de adopción, no solamente para las obras de conservación de suelo y agua, sino para diversificación de la parcela, y para la implementación de estrategias productivas que permitan elevar la calidad de vida de las familias.
En un estudio realizado en México, sobre el Sociopsicoanálisis del campesino mexicano por Fromm, E; Maccoby (1990) encontraron que: aunque la agricultura es la ocupación principal del pueblo y la fuente de subsistencia, la mayoría de las familias $(53 \%)$ no tienen tierra. Los resultados de este estudio, son más ajustados a la realidad de Las Perlas que la propuesta de Mansky, , porque también en la comunidad a pesar de que la agricultura es el motor principal de la economía rural comunitaria, el $60 \%$ de las familias productoras están desprovistas de este medio de producción.

\section{Acceso a mercados}

Uno de los factores que inciden para que las familias rurales puedan mejorar sus condiciones y calidad de vida es el acceso a los mercados y por tanto las oportunidades de ubicar sus productos en estos espacios.

Figura 5. Porcentaje de acceso a mercados.

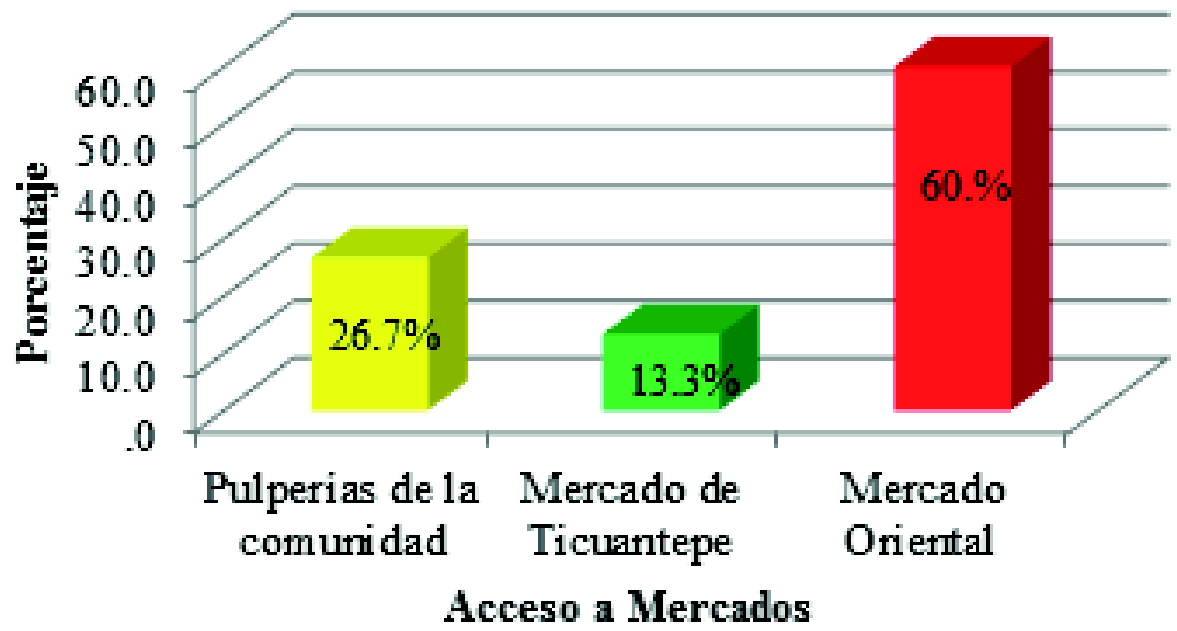

Fuente propia 
El 26.7\% de los productores de Las Perlas comercializan sus productos agrícolas en las pulperías de la comunidad, en este sentido la comunidad es el espacio de mercado local, un $13.3 \%$ comercializan sus rubros agrícolas en el mercado de Ticuantepe (casco urbano), significando que en relación al primero hay preferencia por ubicar los productos en el mercado.

El 60\% de los productores comercializan en el mercado oriental sus productos agrícolas, los productores ven en este espacio de mercado una oportunidad para vender. Este mercado es un contexto de referencia nacional y los productores de la comunidad prefieren ubicar sus productos en el oriental porque hay un mayor número de compradores, y se puede decir que es una venta segura.

Cuando se hace referencia a los espacios de mercado, los productores operan en tres eslabones de una cadena, primero a nivel comunitario (comarca) posteriormente en el casco urbano (Ticuantepe) y por último la capital. La producción de mercancías se mueve en estos tres contextos y tiene un orden de importancia para las y los productores de la comunidad.

En lo referido a la comercialización (Chavarría, 2013) argumenta: "Algunos productores venden sus rubros en Managua, en los mercados: Mayoreo, Oriental, pero la mayoría es para el autoconsumo". Como se refleja en la cita del informante, los principales mercados que tienen los productores, son los que están ubicados en Managua, entre estos está el mercado Oriental, considerado como el más significativo para los productores. Esto se debe a la magnitud de personas que se mueven dentro del este espacio de mercado. Aunque la producción es para garantizar el autoconsumo de las familias. El principal espacio de mercado es el Oriental, pero señala un fenómeno interesante y es la llegada de intermediarios a la comunidad; entonces se está ante la presencia de un nuevo sujeto económico, que se manifiesta de manera esporádica, principalmente en los períodos de cosecha, y que transforma la dinámica cotidiana en cuanto a la comercialización se refiere.

En un análisis hecho por Wolf, E (1971) señala que: el sector campesino llega a estar muy entregado a las operaciones de compra venta a través de la red de mercados y cada vez depende más de los precios que rigen en tales mercados, puede ser afectado incluso por pequeñas fluctuaciones de esos precios. El sistema de compra venta penetra en la comunidad y transforma todas las relaciones en simples tratos de interés mantenidos por los individuos respecto a los productos para la venta.

Se está de acuerdo con el autor que el mercado transforma las relaciones comunitarias, en donde las aspiraciones y el interés principal es vender, el $100 \%$ de los productores de la comunidad comercializan sus productos, que puede ser el excedente en el caso de que cultive granos básicos o la totalidad de la producción en el caso de cultivadores de la piña.

También se coincide que la economía comunitaria y en particular los ingresos familiares dependen del precio de los rubros y estos son definidos por el mercado, se está a expensas de las fluctuaciones de los precios, por lo tanto en los productores prevalece una visión más economicista que social o medioambiental.

Hay un interés particular por obtener la máxima rentabilidad económica, y esto va convirtiendo las relaciones en utilitarias más que fraternas y solidarias, y la economía se fundamenta más en los valores de cambio que en los valores de uso, por tal razón la mayoría de los productores cultivan piña, sin tomar en cuenta el grave daño ambiental que se genera, porque este rubro necesita bastante sol y para eso se requiere talar los recursos forestales. Además con el monocultivo de la piña se vulnera la seguridad alimentaria 
de las familias, porque los productores tienden a cultivar la piña que los sistemas granos básicos y cultivos de patio debido a la diferencia en la rentabilidad, de manera que importa más el ingreso que se pueda percibir que la conservación del ambiente y la alimentación familiar. Las relaciones comunitarias se han transformado en relaciones mercantiles, pero el asunto no es tanto que el productor o productora pueda ubicar sus productos en el mercado, sino que se individualiza.

\section{Conclusiones}

El $86.7 \%$ de las familias rurales consultadas tienen educación primaria y secundaria y un $6.7 \%$ educación técnica, esta realidad es un potencial que permite el desarrollo rural de la comunidad, e indica que hay interés de la familias por ingresar al sistema educativo, a la vez que existe un sistema que da apertura a la formación de estas familias. Un segmento de $6.7 \%$ no saben leer, ni escribir. El $93.4 \%$ de las familias están visualizando la educación en el marco de sus estrategias de desarrollo, si continúa esta tendencia la condición socioeconómica y cultural de las familias se va a transformar en pro del desarrollo comunitario, porque a medida que se avanza en educación las posibilidades de mejorar las condiciones de vida son mayores.

Es importante subrayar en el marco de esta conclusión que si los comunitarios que busquen que tengan formación profesional, buscan oportunidades fuera de la comunidad, particularmente en contextos urbanos, se desencadene en un proceso de descampesinización.

La cultura productiva en la comunidad tiene fortaleza porque el $66.7 \%$ de las familias consultadas trabajan todavía en la agricultura, esta es una actividad económica primaria característica de contextos rurales, aunque un $33.3 \%$ que constituyen comerciantes y trabajadores del seguridad privada $(\mathrm{CPF})$ forman parte de la llamada tercerización de la economía que constituyen actividades extra agrícolas, de manera que la producción agrícola es un aspecto identitario que forman parte la cultura comunitaria rural.

El $53 \%$ de las familias productoras de la comunidad tiene ingresos de 3,000 córdobas mensuales, por debajo del promedio general del salario mínimo establecido a nivel de país, que son 3,448 córdobas para todos los sectores en general, esto no les permite tener apropiadas condiciones de vida.

Por otroladoel $60 \%$ delas familias consultadas no son dueñas de la tierra, esto constituye un factor socioeconómico que obstaculiza el desarrollo rural de la comunidad, porque este es el factor de producción más importante de acuerdo a lo expresado por los comunitarios. El 40\% de las familias son dueños de la tierra, pero hay otro fenómeno que se vincula a esta realidad y es que las mujeres no son dueñas de la tierra, por lo tanto no tienen acceso a fuentes de financiamiento para el estímulo y desarrollo de la producción agrícola.

\section{Referencias bibliográficas}

Alcaldía Municipal de Ticuantepe.2012. Caracterización Municipal: Período 2009-2012. Ticuantepe. s.e.

Basaldúa, M. 2000. Transformación cultural y transformación económica en los campesinos del bajío mexicano. Red de bibliotecas virtuales de ciencias sociales de América Latina y el Caribe. 2000: 1-6. Disponible en http://www. clacso.org.ar/biblioteca

Bonatti, M. 2007. Cambios climáticos, percepciones humanas y desarrollo rural. Tesis Mg en Desarrollo Rural. Buenos Aires. AR. Escuela para graduados Ing. Agr. Alberto Soriano.

Canales, Alvarado y Pineda. 2006. Metodología de Investigación. OPS.

Cino, DM. 2009. Desarrollo Rural Social y Económico: Una experiencia con la introducción del Búfalo en la empresa 
pecuaria MACUN en Villa Clara. Tesis Msc En Desarrollo Social. La Habana, CU. Facultad Latinoamericana de Ciencias Sociales-FLACSO-CUBA.

CIPRES (Centro para la Investigación, la Promoción y el Desarrollo Rural y Social).2006a. Los Pequeños y Medianos Productores Agropecuarios en Nicaragua, Managua, CIPRES Tomo 1.

De Janvry y Sadoulet. 2000. Cómo Transformar en un Buen Negocio la Inversión en el Campesinado Pobre: Nuevas Perspectivas de Desarrollo Rural en América Latina, Nueva Orleans. US.S.E.

Del Ricón, D; Arnal, G; Latorre, A; Sans, A. 1995. Técnicas de Investigación en Ciencias Sociales. Madrid. ES. DYKINSON.

Díaz, T. 2,000. La cultura como factor estratégico del desarrollo rural. Revista de Educación.

Jaramillo, LF. 2007. Elementos para el análisis de la población rural en la zona centro de Urabá: El caso de las comunidades Puerto Girón y Zungo, arriba en Apartadó y Casanova en Turbo. Tesis Mg En Desarrollo Rural, Bogotá, CO, Pontificia Universidad Javeriana.

Feito, MC.2005. Antropología y Desarrollo Rural: Contribuciones del abordaje etnográfico a los procesos de producción e implementación de políticas. REDALYC. no 6:1-26. Disponible en http://www.redalyc. org/

Fonte, M; Acampora, T; Sacco, V. 2006. Desarrollo rural e identidad cultural: Reflexiones teoricas y campos empíricos. Nápoles. IT. RIMISP.

Fromm, E; Maccoby, M.1990. Sociopsicoanálisis del campesino mexicano. México DF. Quinta reimpresión, Fondo de Cultura Económica.

Hernández, R; Fernández, C; Baptista, P.2006. Metodología de la Investigación. México DF. The McGraw-Hill/ Interamericana Editores S.A DE .CV.

Matijasevic, MT. 2007.Significados atribuidos al desarrollo rural en la Vereda, Alto de Zarzo del municipio de Manizales. Tesis Mag en Desarrollo Rural, Bogotá, CO. Pontificia Universidad Javeriana.

Manski, C.F. 1997. The structure of Random Utility Models; Teoría para la toma de decisiones en la adopción. S.E.

Munch, L. 1996. Métodos y Técnicas de Investigación. México DF. Editorial Trillas.

Piura, J. 2006. Metodología de la Investigación Científica, Un enfoque integrador. Managua, NI Ed PAVSA.

Rojas, J; Ramírez, J.2011. Desarrollo Rural en Nicaragua: Una visión de sus problemas y alternativas. Nicaragua. Aldea.

; Espinoza, E. 2013. Desarrollo Rural Territorial: Enfoques, metodologías y experiencias. Nicaragua. Red de Gestión del Conocimiento para el Desarrollo Rural de Matagalpa y Jinotega-SERIDAR.

Romero, J; Lobato, L; Espinoza, V.2009. Ticuantepe y su Historia. Managua. NI. ARDISA.

Rodríguez, G; Gil, J; García, E.1996. Metodología de la investigación Cualitativa. Málaga. ES. Aljibe S.L.

Serra, L.1991. El movimiento campesino: Su participación política durante la revolución popular Sandinista, 19791989. Managua, NI. UCA.

Terry, JR. 2011. Cultura, identidad cultural, patrimonio y desarrollo comunitario rural: una nueva mirada en el contexto del siglo XXI latinoamericano. En Contribuciones a las Ciencias Sociales, no 12. Disponible en: www.eumed. net/rev/ccss/12/

Vargas, O. 2005. La Educación en Nicaragua: 1990-2005. Managua. NI. EDITARTE.

Wolf, E.1971. Los campesinos. Barcelona, ES Labor S.A. 149 p.

Zúniga, C.2011. Texto básico de Economía Agrícola: Su importancia para el desarrollo local sostenible.LeónNicaragua. Editorial Universitaria UNAN-León. 